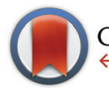

CrossMark \&click for updates

Cite this: Polym. Chem., 2015, 6, 7945

Received 14th August 2015,

Accepted 22nd September 2015

DOI: $10.1039 /$ c5py01293g

www.rsc.org/polymers

\section{Improved control through a semi-batch process in RAFT-mediated polymerization utilizing relatively poor leaving groups $\uparrow$}

\author{
Alexander Ilchev, Rueben Pfukwa, Lebohang Hlalele, Marica Smit and \\ Bert Klumperman*
}

\begin{abstract}
The negative effect that a RAFT agent with a poor leaving group has on the evolution of molecular dispersity in a RAFT-mediated polymerization was shown to be mitigated by performing the polymerization in semi-batch mode. The result is attributed to an increase in the probability of transfer between the propagating radical and the leaving group during the polymerization. Also for RAFT-mediated polymerizations that use RAFT agents with efficient leaving groups, the evolution of molecular dispersity during a semi-batch polymerization improves compared to that for an analogous batch-mode reaction.
\end{abstract}

\section{Introduction}

Radical polymerization is one of the most commonly used methods for synthesizing commercial polymers since it is a robust method to prepare polymeric materials. ${ }^{1-3} \mathrm{~A}$ high tolerance to impurities and many different functional groups makes radical polymerization preferable over ionic polymerization for industrial processes. Reaction temperatures also play a less crucial role for radical polymerization than for ionic polymerization. However, there are drawbacks to conventional radical polymerization. Most notably, there is a characteristically poor control over the molecular weight distribution. ${ }^{2-4}$ Over the past two decades, techniques have been developed that are collectively referred to as Reversible Deactivation Radical Polymerization (RDRP) that overcome the drawbacks mentioned above. $^{5-10}$ Arguably, among the most efficient of the RDRP techniques is RAFT (Reversible Addition-Fragmentation chain Transfer) mediated polymerization. RAFT-mediated polymerization is compatible with virtually all monomer classes that are accessible via conventional radical polymerization. Important to note is that the mediating thiocarbonyl thio compound (RAFT agent) must be tuned in terms of reactivity to the

Department of Chemistry and Polymer Science, Stellenbosch University, Private Bag X1, Matieland 7602, South Africa.E-mail: bklump@sun.ac.za

$\dagger$ Electronic supplementary information (ESI) available: Detailed experimental protocols and additional SEC data. See DOI: 10.1039/c5py01293g specific monomer being polymerized. ${ }^{11-13}$ For example, several researchers have referred to more activated monomers (MAMs) such as styrene and acrylates and less activated monomers (LAMs) such as vinyl acetate and $N$-vinylpyrrolidone (NVP). Where MAMs are typically mediated by dithiobenzoates and trithiocarbonates, ${ }^{14-16}$ the polymerization of LAMs requires dithiocarbamates ${ }^{17,18}$ or xanthates ${ }^{16,19-21}$ for good control. In addition, earlier work from our group ${ }^{19}$ and others has shown that the choice of the so-called leaving group (or R-group) is also essential in obtaining good control. It is commonly known that the choice of a RAFT agent with a large chain transfer constant is essential to obtain a narrow molar mass distribution. However, the factor that controls the degree of control is the probability of chain transfer. It has earlier been pointed out by Moad and co-workers that in a polymerization controlled via degenerative chain transfer, apart from a chain transfer agent (CTA) with a large chain transfer constant, also a low ratio of the monomer concentration to the CTA concentration will lead to a large probability of chain transfer and therefore a narrow molar mass distribution. ${ }^{6,22,23}$ Methacrylic macromonomers are relatively poor CTAs, which Moad and coworkers used to mainly study the synthesis of block copolymers. In starved feed emulsion polymerization experiments they reach dispersities $(\theta)$ as low as $1.2-1.3 .^{23}$ Similar experiments conducted in solution show values of $D \cong 1.5 .^{22}$ The manipulation of the monomer-to-RAFT agent ratio to improve control over the polymerization can be used to address either the R- or Z-group effect of the RAFT agent. In the event of a relatively poor Z-group, as in the case of a xanthate-mediated polymerization of MAMs, a continuous slow addition of monomer can overcome the inherently low $C_{\mathrm{tr}}$, maximizing the RAFT-to-monomer ratio at any instant during the polymerization. Monteiro and coworkers later confirmed the use of slow monomer addition to improve the level of control (as judged by a low $D$ ) in a RAFT-mediated emulsion polymerization utilizing a xanthate as a chain transfer agent. ${ }^{24,25}$ However, in the event of a relatively poor R-group, a discrete semi-batch process can lead to improved $D$ values, in contrast to the case of a poor Z-group which requires a continuous semi-batch 


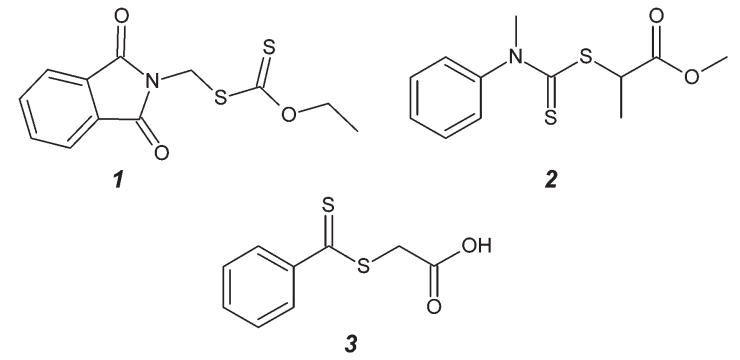

Fig. 1 Structures of RAFT agents employed in the batch and semibatch polymerizations.

process. Surprisingly, this effect has, to the best of our knowledge, never been systematically investigated using typical RAFT agents such as dithiobenzoates, xanthates, and dithiocarbamates for a solution polymerization. As such, the focus of the current study is to demonstrate the versatility of RAFT agents considered to bear a relatively poor leaving group, by improving the $Ð$ value through the adjustment of reaction conditions to the use of a semi-batch process.

In the present contribution we will show examples of RAFTagent/monomer combinations that provide poor control when conducted as a batch polymerization $(\nexists \cong 1.5)$. We will then show that the degree of control can be significantly improved in some cases by performing the reaction in semi-batch mode. The first example will be the RAFT-mediated polymerization of NVP in the presence of $O$-ethyl-S-(phthalimidylmethyl)xanthate (RAFT agent 1 - Fig. 1), which was used previously to provide poly( $N$-vinylpyrrolidone) (PVP) with an amine-functionalized $\alpha$-end group (after deprotection). ${ }^{16}$

\section{Results and discussion}

As was pointed out earlier by Moad and coworkers, the probability of chain transfer can be approximated by eqn (1):

$$
p_{\mathrm{tr}}=\frac{R_{\mathrm{tr}}}{R_{\mathrm{tr}}+R_{\mathrm{p}}}=\frac{k_{\mathrm{tr}}[\mathrm{RAFT}]}{k_{\mathrm{tr}}[\mathrm{RAFT}]+k_{\mathrm{p}}[\mathrm{M}]}=\frac{C_{\mathrm{tr}}}{C_{\mathrm{tr}}+[\mathrm{M}] /[\mathrm{RAFT}]}
$$

For a RAFT-mediated polymerization, the equilibrium constant for chain transfer to the initial RAFT agent is defined as a composite term, shown in eqn (2). This, however, has no effect on the general applicability of eqn (1):

$$
C_{\mathrm{tr}}=\frac{k_{\mathrm{tr}}}{k_{\mathrm{p}}}=\frac{k_{\mathrm{add}}}{k_{\mathrm{p}}} \times \frac{k_{\beta}}{k_{-\mathrm{add}}+k_{\beta}}
$$

The general strategy to obtain good control (i.e. low $\boxplus$ ) in a RAFT-mediated polymerization is by the use of RAFT agents with a high chain transfer constant $C_{\mathrm{tr}}=k_{\mathrm{tr}} / k_{\mathrm{p}}$, which leads to a large probability of chain transfer. However, inspection of eqn (1) leads to the conclusion that an alternative strategy to obtain good control is the selection of a low ratio of monomer concentration to RAFT agent concentration ([M]/[RAFT $]$ ).

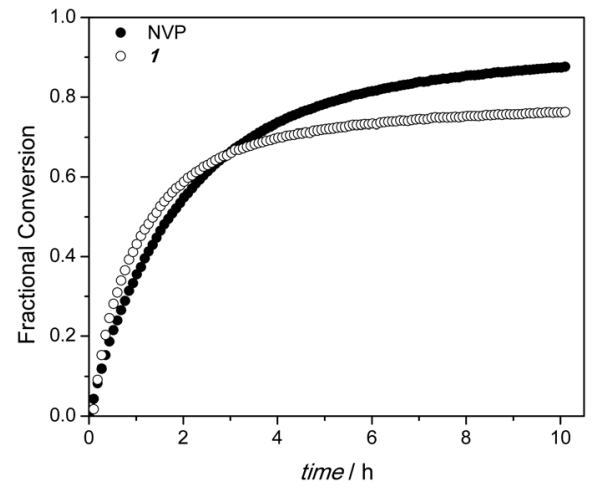

Fig. 2 Fractional conversion profiles for 1 and NVP monitored by in situ ${ }^{1} \mathrm{H}$ NMR during a polymerization carried out at $65^{\circ} \mathrm{C}$ with [NVP] : [1] : $[\mathrm{AIBN}]=5: 1: 0.1$.

In order to test the efficacy of RAFT agent $\mathbf{1}$ as a RAFT agent for the polymerization of NVP, an initialization experiment was conducted according to the procedure described in the ESI. $\dagger$ Fig. 2 shows the fractional conversion profiles of NVP and RAFT agent 1.

Fig. 2 clearly shows that the RAFT agent does not get fully converted into the macro-RAFT agent. The most plausible explanation for this behaviour is that the oligo-NVP chains are better leaving groups than the original phthalimidomethyl leaving group. In other words, RAFT agent 1 possesses a low chain transfer constant.

Next, two batch-wise RAFT agent 1-mediated NVP polymerizations were carried out in which two different monomer-toRAFT agent ratios were used, i.e. 117 and 196 (entries 1 and 2, Table 1). Samples were taken from the reaction mixture at various stages (Tables 1 and $\mathrm{S} 1 \dagger$ ). Two important observations can be made. First, the conversion of the RAFT agent into macro-RAFT agent only occurs very gradually, and even at the end of the experiments, small amounts of the original RAFT agent are still present (Table S1†).

The evolution of molecular weight and dispersity with increasing monomer conversion for target DP of 117 and 196 is shown in Fig. 3. Second, the dispersity values of the polymers are consistently around 1.5 throughout the polymerizations, in agreement with results previously reported by Postma et al. ${ }^{16}$ This is a frequently observed phenomenon that is particularly common for polymerizations in which RAFT agents with a relatively low chain transfer constant are employed.

To overcome the effect of a low chain transfer constant, polymerizations were carried out in a semi-batch mode. The essence is that the monomer is fed in stages into the reaction. As a consequence, the initial monomer to RAFT agent ratio is low compared to a batch reaction, and similar degrees of polymerization can still be reached. In the case where NVP polymerization was mediated with RAFT agent $\mathbf{1}$, the monomer was fed in a stepwise fashion instead of utilizing a continuous feed. The stepwise additions were performed in 
Table 1 Experimental results for RAFT-mediated polymerization of $N$-vinylpyrrolidone, styrene and methyl methacrylate in batch and semi-batch mode

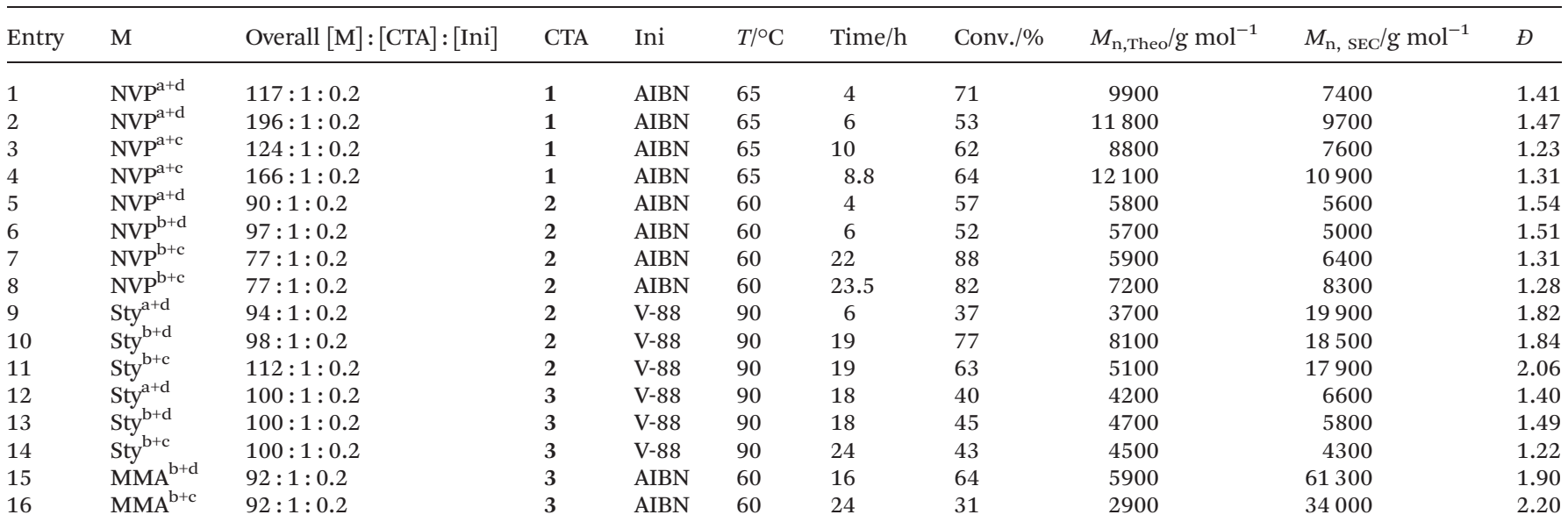

${ }^{\mathrm{a}}=$ bulk polymerization, ${ }^{\mathrm{b}}=$ solution polymerization, ${ }^{\mathrm{c}}=$ semi-batch mode, ${ }^{\mathrm{d}}=$ batch mode .

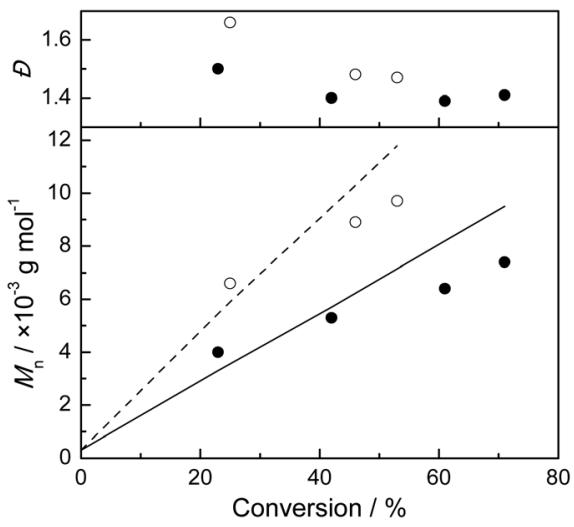

Fig. 3 Evolution of $M_{\mathrm{n}}$ and $\Theta$ with conversion for NVP polymerization in batch mode, using RAFT agent 1 . The employed monomer to RAFT agent ratios are $117(\bullet)$ and $196(0)$, entries 1 and 2 in Table 1, respectively.

such a way that the monomer conversion was kept around $60 \%$. Increasing the conversion to even higher values would lead to a larger probability of termination reactions, which have been neglected in eqn (1). Tables S3 and S5† show the results of two semi-batch experiments where the target degrees of polymerization were 124 and 166, respectively. The final dispersity values for the target degree of polymerization of 124 and 166 are shown in Table 1, entries 3 and 4, respectively. Compared to the batch experiments, it is immediately clear that the conversion of the RAFT agent is much larger already at early stages of the polymerization and reaches full conversion well before the end of the experiment (Tables S3 and $\mathrm{S} 5 \dagger)$. Simultaneously, the dispersities are relatively low from the early stages of the reaction. Fig. 4 shows the evolution of $M_{\mathrm{n}}$ and $D$ for a polymerization carried out in a semi-batch mode, for an overall target degree of polymerization of 124

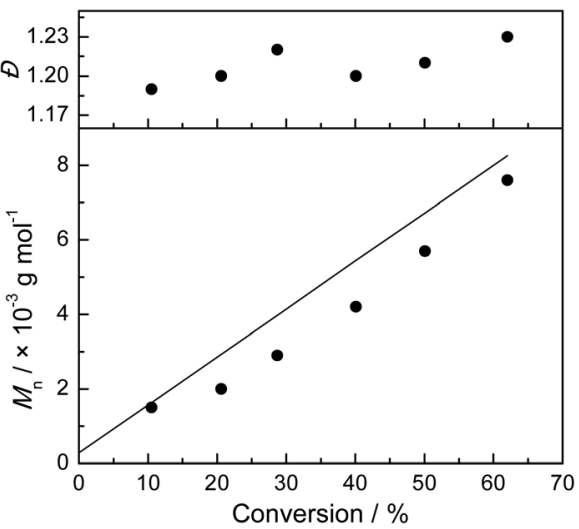

Fig. 4 Evolution of $M_{n}$ and $\oslash$ as a function of conversion for NVP polymerization in semi-batch mode, using RAFT agent 1 (entry 3 , Table 1).

(entry 3, Table 1). The monomer conversion values in Fig. 4 are based on the overall monomer used, and the theoretical molar masses are calculated based on the overall monomerto-RAFT ratio.

A better correlation between expected and measured number average molecular weight values is seen in Fig. 4 (semi-batch mode polymerization) compared to Fig. 3 (batch mode polymerization). Further comparisons between batch and semi-batch mode of polymerization are exemplified in Table 1 (and Tables S2-S11†) for various RAFT agent/monomer combinations.

In cases where batch polymerization yields polymers with $D>1.6$, a switch to semi-batch mode of polymerization does not improve the control over the polymerization. This is clearly evidenced in cases of polymerizations of styrene and methyl methacrylate mediated by RAFT agents 2 (entries 9-11, Table 1) and 3 (entries 15 and 16, Table 1), respectively. 
Previously reported batch polymerizations of styrene and MMA in solution mediated by RAFT agent 3 exhibited dispersity values in agreement with those obtained in this study. ${ }^{26}$ In the case of MMA polymerization, it can be postulated that the oligo-MMA tertiary radical is a far better leaving group than the R-group of RAFT agent 3, thereby affording no improvement by switching to the semi-batch mode of polymerization. The improved control, obtained by switching to a semi-batch polymerization process, is significant in that it eliminates the need for one to use RAFT agents with excellent leaving groups, which often require demanding synthetic protocols.

\section{Conclusions}

Control over RAFT mediated polymerizations was improved by performing the experiment in semi-batch mode. In doing so, the ratio of the monomer concentration to the RAFT agent concentration is kept low at early stages of the polymerization. This directly increases the probability of chain transfer and therefore mitigates the negative effect of a low chain transfer constant on the width of the molar mass distribution. Our preliminary assessment on employing the concept of varying monomer-to-RAFT agent ratio to improve control is that such a protocol is suited for systems in which the batch process yields $Ð \cong 1.5$.

\section{Acknowledgements}

The authors acknowledge the support through the South African Research Chairs Initiative (SARChI) from the Department of Science and Technology (DST) and the National Research Foundation (NRF) of South Africa. BK and $\mathrm{LH}$ acknowledge financial support from Subcommittee B of Stellenbosch University.

\section{References}

1 P. Nesvadba, Radical Polymerization in Industry; Encyclopedia of Radicals in Chemistry, Biology and Materials, John Wiley \& Sons Ltd, 2012.

2 K. Matyjaszewski and T. P. Davis, Handbook of Radical Polymerization, John Wiley and Sons Inc., Hoboken, New Jersey, 2002.

3 G. Odian, Principles of Polymerization, John Wiley \& Sons, Inc., Hoboken, New Jersey, 2004.

4 K. Yoo and H. Rhee, AIChE J., 1999, 45, 1298-1308.

5 C. Barner-Kowollik, Handbook of RAFT Polymerization, Wiley-VCH Verlag GmbH \& Co. KGaA, Weinheim, Baden-Württemberg, 2008.
6 G. Moad, J. Chiefari, Y. K. Chong, J. Krstina, R. T. A. Mayadunne, A. Postma, E. Rizzardo and S. H. Thang, Polym. Int., 2000, 49, 993-1001.

7 G. Moad, E. Rizzardo and S. H. Thang, Aust. J. Chem., 2005, 58, 379-410.

8 R. B. Grubbs, Polym. Rev., 2011, 51, 104-137.

9 K. Matyjaszewski, Macromolecules, 2012, 45, 40154039.

10 J. Chiefari, Y. K. B. Chong, F. Ercole, J. Krstina, J. Jeffery, T. P. T. Le, R. T. A. Mayadunne, G. F. Meijs, C. L. Moad, G. Moad, E. Rizzardo and S. H. Thang, Macromolecules, 1998, 31, 5559-5562.

11 J. Chiefari, R. T. A. Mayadunne, C. L. Moad, G. Moad, E. Rizzardo, A. Postma, M. A. Skidmore and S. H. Thang, Macromolecules, 2003, 36, 2273-2283.

12 Y. K. Chong, J. Krstina, T. P. T. Le, G. Moad, A. Postma, E. Rizzardo and S. H. Thang, Macromolecules, 2003, 36, 2256-2272.

13 N. Akeroyd, R. Pfukwa and B. Klumperman, Macromolecules, 2009, 42, 3014-3018.

14 R. T. A. Mayadunne, E. Rizzardo, J. Chiefari, J. Krstina, G. Moad, A. Postma and S. H. Thang, Macromolecules, 2000, 33, 243-245.

15 A. Postma, T. P. Davis, R. A. Evans, G. Li, G. Moad and M. S. O'Shea, Macromolecules, 2006, 39, 5293-5306.

16 A. Postma, T. P. Davis, G. Li, G. Moad and M. S. O'Shea, Macromolecules, 2006, 39, 5307-5318.

17 D. J. Keddie, C. Guerrero-Sanchez, G. Moad, R. J. Mulder, E. Rizzardo and S. H. Thang, Macromolecules, 2012, 45, 4205-4215.

18 M. Destarac, D. Charmot, X. Franck and S. Z. Zard, Macromol. Rapid Commun., 2000, 21, 1035-1039.

19 G. Pound, J. B. McLeary, J. M. McKenzie, R. F. M. Lange and B. Klumperman, Macromolecules, 2006, 39, 77967797.

20 G. Pound, F. Aguesse, J. B. McLeary, R. F. M. Lange and B. Klumperman, Macromolecules, 2007, 40, 88618871.

21 M. H. Stenzel, L. Cummins, G. E. Roberts, T. P. Davis, P. Vana and C. Barner-Kowollik, Macromol. Chem. Phys., 2003, 204, 1160-1168.

22 J. Krstina, C. L. Moad, G. Moad, E. Rizzardo and C. T. Berge, Macromol. Symp., 1996, 111, 13-23.

23 J. Krstina, G. Moad, E. Rizzardo, C. L. Winzor, C. T. Berge and M. Fryd, Macromolecules, 1995, 28(15), 5381-5385.

24 M. J. Monteiro and J. d. Barbeyrac, Macromolecules, 2001, 34, 4416-4423.

25 W. Smulders and M. J. Monteiro, Macromolecules, 2004, 37, 4474-4483.

26 S. C. Farmer and T. E. Patten, J. Polym. Sci., Part A: Polym. Chem., 2002, 40, 555-536. 